\title{
HISTORISCHE GÄRTEN IM KLIMAWANDEL
}

\author{
Erkenntnisse, Desiderata und Empfehlungen'
}

\section{Präambel}

Historische Gärten, Parks und Kulturlandschaften sind denkmalwerte Kulturgüter und ebenso wie Bauwerke und Kunstsammlungen unwiederbringliche Zeugnisse unserer Zivilisation. Daher werden sie geschützt, gepflegt und erforscht, um sie weiterhin für die Gesellschaft erhalten zu können. Durch die weltweit wahrnehmbaren Klimaveränderungen sind sie inzwischen in besonderem Maße gefährdet. Die Mitglieder der interdisziplinären Arbeitsgruppe (IAG) »Historische Gärten im Klimawandel« der Berlin-Brandenburgischen Akademie der Wissenschaften haben sich zwischen 2016 und 2019 der Frage gewidmet, ob und auf welche Weise für ihre Bewahrung Vorsorge getroffen werden kann. Historische Gärten bilden die Auswirkungen des Klimawandels auf überschaubarem Raum insofern verdichtet ab, als sie dessen Folgen für Kultur, Natur und Gesellschaft gleichermaßen spiegeln. Sie eignen sich daher in besonderer Weise für eine vergleichende Analyse von Optionen der Anpassung an sich wandelnde Bedingungen.

Während historische Gärten schon seit vielen Jahren Gegenstand der Denkmalpflege und der (Garten-)Kunstgeschichte sind, haben in der IAG erstmals zusätzlich zu diesen Disziplinen Natur- und Sozialwissenschaftlerinnen und -wissenschaftler gemeinsam an vier Beispielgärten geforscht. Das heißt, es wurde erstmals systematisch die denkmalpflegerische und kunsthistorische Perspektive um eine naturräumliche Sicht auf historische Gärten und um eine Untersuchung ihrer Rolle als Räume sozialen Handelns erweitert. Diese interdisziplinäre Perspektive war nicht nur ein ungeheurer Gewinn für die beteiligten Wissenschaftlerinnen und Wissenschaftler selbst, sondern führte darüber hinaus zu einem erheblichen Erkenntnisgewinn im Hinblick auf eine Entwicklung möglicher Strate-

Im Text werden Maskulinum und Femininum verwendet, wenn es um Personen geht. Gemeint sind grundsätzlich alle Menschen, gleich welcher Geschlechtsidentität sie sich zugehörig fühlen.

1 Dieses Kapitel ist das Ergebnis mehrerer Diskussionen der Mitglieder in den Plenumssitzungen der IAG »Historische Gärten im Klimawandel«. 
gien für die Bewahrung der historischen Gärten unter den Bedingungen des Klimawandels. Allen an der IAG Beteiligten ist im Verlauf der Diskussionen klargeworden, dass nur eine interdisziplinäre Herangehensweise geeignet ist, um die Gärten als schützenswerte Kulturgüter langfristig erhalten zu können.

Der vorliegende Text fasst die Untersuchungen der IAG zusammen und enthält erste Empfehlungen, die sich einerseits an die für die Gärten Verantwortlichen richten, andererseits Anregungen für Politik und Gesellschaft enthalten, um sie für die Funktionen und Werte historischer Gärten, Parks und Kulturlandschaften verstärkt zu sensibilisieren.

Ausgangspunkt der folgenden Überlegungen ist die Annahme, dass sich die Anforderungen an die Gartendenkmalpflege angesichts des Klimawandels verschoben und erweitert haben. Der Wert historischer Gärten als Kunst- und Geschichtszeugnisse begründet den denkmalpflegerischen Auftrag. Die Einzigartigkeit dieser Gärten besteht darin, dass sie zugleich unverrückbare Naturräume sind, die von Seiten der Naturwissenschaften bislang kaum systematisch und umfassend erfasst und beschrieben wurden. Als gesellschaftliche Räume der Geschichte und Gegenwart sind sie darüber hinaus eine gesellschaftliche Projektionsfläche und interessieren daher die Sozialwissenschaften. Ein abschließender Ausblick eröffnet Perspektiven auf künftige Aufgabenfelder für Wissenschaft und Politik.

\section{Warum ist der Klimawandel für historische Gärten von besonderer Relevanz?}

Historische Gärten, Parks und Kulturlandschaften stellen im Kanon der Künste eine Besonderheit dar, da sie neben einer vielfältigen künstlerischen Ausstattung hauptsächlich aus belebten Materialien bestehen, insbesondere aus Bäumen, Sträuchern, Blumen, Rasen, Wiesen, Boden und Gewässern. Aufgrund der Umweltabhängigkeit dieser künstlerisch angeordneten Materialien trifft der derzeitige Klimawandel mit seinen Auswirkungen (Stürme, Starkniederschläge, Hitzeperioden, Temperaturanstieg, veränderte Bodenverhältnisse etc.) diese Kunstwerke weitgehend ungeschützt. Als kulturelle Zeugnisse und wertvoller Bestandteil der menschlichen Zivilisation gilt es jedoch, sie im gesellschaftlichen Auftrag zu bewahren.

Die singuläre Eigenheit historischer Gärten besteht darin, dass sie gleichermaßen Räume und somit auch Systeme der Kultur, der Natur und der Gesellschaft sind. Das heißt, dass sich die klimatischen Veränderungen der Vergangenheit und der Gegenwart in ihnen wie in einem Brennglas gebündelt abbilden. Ihr strukturreicher Aufbau ist einerseits ein inhärenter Teil der (Gesamt-)Kunstwerke und andererseits schafft er vielgestaltige Naturräume mit hoher Biodiversität einschließlich genetischer Vielfalt. Historische Gärten sind zudem sowohl Spiegel vergangener Gesellschaften als auch Aufenthaltsort zur Erholung und Bildung für die heutigen Besucherinnen und Besucher. Diese Funktionsvielfalt historischer Gärten wird durch den aktuellen Klimawandel in ihrer gesamten Breite bedroht.

Aufgabe der Wissenschaften und der Gartendenkmalpflege ist es, angesichts dieser Entwicklung klassische Denkmalkriterien und -funktionen (z. B. künstlerische, geschichtliche, wissenschaftliche, ökonomische, städtebauliche) verstärkt zu vermitteln, neu zu reflektieren und dabei auszuloten, wie daraus gesellschaftliches Identifikationspotential 
entwickelt werden kann. Der Klimawandel und seine Wirkung auf historische Gärten könnten hier eine wichtige Funktion übernehmen, um den Wert der Gärten für die Stiftung kultureller Identitäten in einen neuen Kontext zu stellen.

\section{Anforderungen an die Gartendenkmalpflege}

Die Gartendenkmalpflege ist dafür verantwortlich, den Zustand und das Erscheinungsbild des Gartens im gesellschaftlichen Auftrag zu erhalten. Ihr Wissen und ihre Methodik haben sich seit etwa 100 Jahren in den Gärten selbst entwickelt und überliefert, werden jedoch auch seit ca. 30 Jahren an Universitäten und Fachschulen gelehrt. Denkmalschutzgesetze und internationale Vereinbarungen festigen den gesetzlichen Rahmen und legen die Ausrichtung der Gartendenkmalpflege fest. Die Charta von Florenz (1981) widmet sich explizit historischen Gärten, Parks und Kulturlandschaften. Sie unterstreicht, dass die Authentizität der Gärten in der Planung der ästhetischen und räumlichen Konzeption der verschiedenen Partien, schmückenden Ausstattung, Pflanzenwahl und Baumaterialien besteht. Eine Entscheidung über Nachpflanzungen, wenn beispielsweise die bisherigen Arten aufgrund des Klimawandels nicht mehr gedeihen können, kann nur auf Grundlage des gesammelten praktischen und theoretischen Wissens zur Entstehungsgeschichte, zu den einzelnen im Garten anzutreffenden Zeitschichten und ihrer jeweiligen Bedeutung getroffen werden. Die Gartendenkmalpflege arbeitet stets im Austausch mit dem Naturschutz, denn nicht nur die künstlerischen, sondern auch die naturräumlichen Besonderheiten historischer Gärten drohen mit fortschreitendem Klimawandel geschädigt zu werden oder gar unwiederbringlich verloren zu gehen. Als strukturreiche Landschaftselemente haben sich historische Gärten gerade in urbanen und suburbanen Räumen zu Refugien der biologischen Vielfalt entwickelt. Gleichwohl wird empfohlen, dass dem denkmalpflegerischen Auftrag, soweit die angestrebte Harmonisierung beider Ziele nicht erreicht werden kann, ein Vorrang vor naturschutzfachlichen Maßnahmen eingeräumt wird. Der Erhalt historischer Gärten erfordert im Vergleich zu anderen Kunstgattungen in einem weitaus höheren Maße permanent konservierende Fachpflege wie auch restaurierende Maßnahmen: Hecken sind zu schneiden, Rasen zu mähen, Sichtachsen frei zu halten, Bäume nach Erreichen ihres Lebenszeitalters zu ersetzen etc. Im Jahresgang fallen zudem weitere immer wiederkehrende Aufgaben für die Gärtnerinnen und Gärtner an. Das für dieses breite Spektrum an Pflegemaßnahmen verantwortliche Personal muss entsprechend ausgebildet sein und in ausreichender Anzahl eingestellt werden, wie nationale und internationale Fachkreise bereits seit längerem anmahnen.

Die Empfehlungen zur Bewahrung historischer Gärten als Gesamtkunstwerk werden auf unterschiedlichen Ebenen wirksam und reichen von der denkmalpflegerischen Praxis bis hin zum Diskurs mit relevanten gesellschaftlichen Gruppen und politischen Entscheidungsträgern. Vor diesem Hintergrund ergeben sich aus denkmalpflegerischer Sicht die folgenden Empfehlungen: 
- Der Wert der historischen Gärten wird wesentlich durch die gebaute Infrastruktur mitbestimmt: historische Gebäude, Brücken, Wege, künstliche und natürliche Gewässer, Mauern und Statuen. Gerade diese Strukturen sind durch den Klimawandel zunehmend gefährdet. Um dem denkmalpflegerischen Auftrag gerecht zu werden, ist es höchste Zeit, die Substanz der Bauwerke in den historischen Gärten in einem zu beschließenden Turnus mit hochkarätigen Technologien zu analysieren. Optimal wäre der Einsatz entsprechender Methoden, wenn sich an Bauwerken von sich aus beginnende Mängel zeigen. Dank neuer Sensortechnologien kann anhand der Temperaturverteilung auf der Bauteiloberfläche beispielsweise auf tiefer liegende Anomalien geschlossen werden.

- Mit Blick auf die Rolle neuer - insbesondere digitaler - Technologien zur Prävention, Risikominderung, Beobachtung und Planung erscheint es dringend erforderlich, die Ausbildungsprofile in der Gartendenkmalpflege auf allen Ebenen weiter zu entwickeln. Die IAG liefert hier die Blaupause für einen interdisziplinär strukturierten Studiengang. Zu überlegen wäre ferner, wie die nachgeordneten berufsqualifizierenden Ausbildungsinhalte an diese neuen Rahmenbedingungen angepasst werden können. Damit einhergehend bedarf es dringend auf allen Ebenen einer Anstrengung, um überliefertes Erfahrungswissen zu identifizieren, zu bewerten und verfügbar zu machen.

- Aus der Summe nutzungs- und klimabedingter Schäden resultiert eine Erhöhung des gärtnerischen Arbeitspensums und auch des Aufwandes im organisatorischen Bereich. Daher erscheint eine Verstärkung des gartenpflegerischen Fachpersonals und eine finanzielle Verstärkung im Managementbereich unumgänglich. Für die Kompensation der durch zusätzliches Schadaufkommen entstehenden Verluste müssen darüber hinaus höhere Sachmittelbudgets veranschlagt werden.

\section{Der Zeugnis- und Kunstwert historischer Gärten}

Historische Gärten bestehen maßgeblich aus bedeutungsreichen und Sinn stiftenden Räumen und Bildern. Um die notwendigen Maßnahmen für ihren Erhalt ermitteln zu können, bedarf es grundsätzlich einer Erforschung ihres individuellen Kunst- und Denkmalwertes. Diese Bewertung erfolgt vor dem Hintergrund der Diskussion um Authentizität und Originalität in der Gartenkunst, die aufgrund der stetigen Veränderungen ihrer natürlichen Bestandteile von den übrigen Kunstgattungen abweicht. Insbesondere im Hinblick auf den Spielraum, der zwischen Erhalt und Wiederherstellung besteht, sind spezifische Maßstäbe anzulegen.

Gärten als begehbare Kunstwerke besitzen stets eine künstlerisch ausgestaltete, auf den Menschen und seine Eigenbewegung (Kinästhetik) bezogene, anschaulich beschreibbare Topographie. Diese wird insbesondere durch Geländemodellierungen, Gewässerpartien und Vegetationsanordnungen erreicht. Bauten, Statuen, Grotten und Inschriften werden 
über Wegführungen erschlossen und zeichnen so einen programmatischen Bild- und Sinnzusammenhang (Ikonologie).

Wenn nun aber beispielsweise Bäume, die wesentlich für eine Gartenpartie oder eines der Gartenbilder sind, allmählich absterben oder umstürzen, geht der durch Authentizität und Originalität definierte Kunstwert verloren. Dies mindert die kulturelle Bedeutung und den Bildungswert des Gartendenkmals empfindlich.

Über Einordnungen und Wertungen der formalen Gestaltung im Kontext der Gartenkunstgeschichte und Theorie der Gartenkunst ist der Stellenwert der jeweiligen Anlage individuell zu ermitteln, was für herausragende Gärten bereits weitgehend geleistet ist (z.B. UNESCO-Welterbeliste, Parkpflegewerke). Angesichts der schon eingetretenen oder sich abzeichnenden vermehrten Schädigungen und Verluste durch den Klimawandel gewinnt diese Aufgabe an Dringlichkeit. Es ist essenziell, Öffentlichkeit und Politik von der wichtigen kulturellen Bedeutung dieser Gärten zu überzeugen, um Maßnahmen der Gartendenkmalpflege begründen und überhaupt auf den Weg bringen zu können.

Gegründet auf dem Methodenkanon der Gartenkunstgeschichte können ursprüngliche Gestaltungsphasen rekonstruiert werden, das heißt anhand von Quellenmaterial wie Bestandsplänen, Schriften und Archivalien, aber auch mittels bodenarchäologischer Untersuchungen. Eine Analyse beispielsweise des Bestands an Pflanzungen, Wasseranlagen, Wegen, des Umgangs mit der Topographie und der architektonischen Ausstattung erlaubt wissenschaftliche Aussagen zu den intendierten künstlerischen Wirkungen, die jeweils für bestimmte Regionen, Kulturen, Epochen etc. charakteristisch sind.

Die Ermittlung dieser künstlerischen und historischen Zeugniswerte ist allen Maßnahmen grundsätzlich voranzustellen, denn:

- Auf den Expertisen der Gartenkunstgeschichte und Denkmalpflege basieren Entscheidungen hinsichtlich der Elemente, die in dem jeweiligen historischen Garten, Park oder einer Kulturlandschaft unbedingt erhalten oder wiederhergestellt werden müssen.

- Angesichts der Vielzahl von eingetragenen Gartendenkmalen in Deutschland ist es dringend anzuraten, eine solche Beschreibung und Wertung generell allen gartenpraktischen Maßnahmen voranzustellen.

- Maßnahmenkataloge für die Gartendenkmalpflege können allerdings nur unter Berücksichtigung der naturräumlichen Handlungsspielräume entworfen werden, die von den Naturwissenschaften zu ermitteln sind (s.u.).

- Informationen zum Umgang mit Klima und Wetter in der Vergangenheit müssen dazu systematisch erschlossen und quellenkritisch ausgewertet und den Naturwissenschaften zur Verfügung gestellt werden.

- Basierend auf der Auswertung historischer Dokumente aus der Doppelperspektive der Geistes- mit Naturwissenschaften ist die Diskussion über den Spielraum von Anpassungsmaßnahmen angesichts der Klimaszenarien neu aufzustellen. 
- Künftig sollte das gesamte Spektrum der seit ihrer Entstehung in historischen Gärten eingegangenen Expertisen sowie des Erfahrungswissens der Gärtner wieder stärker zusammengeführt werden.

\section{Historische Gärten als spezifische Naturräume}

Historische Gärten stellen als Habitate, ausgestattet mit heimischen und fremdländischen Arten an Gehölzen, eine eigene Nutzungskategorie dar, deren Funktionsweise, Dynamik und Nutzwirkungen bisher im Unterschied zu anderen Kulturlandschaften naturwissenschaftlich wenig erforscht sind. Erst seit den 1980er Jahren sind historische Gärten verstärkt zum Gegenstand naturwissenschaftlicher Forschung geworden. Diese beschränkte sich zunächst überwiegend auf die Analyse der Vielfalt von Fauna und Flora. Mit der Entwicklung ökosystemarer Forschungsansätze stieg die Erkenntnis, dass naturnahe Systeme durch ein komplexes Wirkungsgefüge vielfältiger biotischer und abiotischer Faktoren (Boden, Wasser, Vegetation, Fauna, Klima, Mensch) gekennzeichnet sind, die generellen Gesetzmäßigkeiten folgen, aber standortspezifisch sehr unterschiedlich ausgeprägt sein können. Der sich vollziehende Klimawandel greift aktuell tief in dieses Wirkungsgefüge ein. Geeignete Maßnahmen zum Schutz historischer Gärten können daher nur gelingen, wenn sie die jeweiligen standörtlichen Bedingungen berücksichtigen. Der Mangel an Informationen zu den spezifischen Standorteigenschaften stellt daher den denkmalpflegerischen Auftrag gerade im Hinblick auf den Klimawandel vor neue Herausforderungen. Erst ein umfassendes Wissen zu den naturräumlichen Gegebenheiten ermöglicht eine bessere Einschätzung gegenüber klimatischem Stress.

Wetter und Klima werden schon lange erforscht. Die aktuellen Vorhersagemodelle prognostizieren eine signifikante Zunahme langer Trockenheitsphasen, sommerlicher Temperaturextreme sowie steigende Temperaturen und veränderte Niederschlagsverhältnisse. Modelle für eine kleinräumig differenzierte Aussage zur Wirkung der Vegetationsstruktur auf das lokale Klima fehlen jedoch bislang. Anhand ausgewählter Beispiele konnte die IAG zeigen, dass die Vegetationsstruktur einen wichtigen Einfluss auf das Mikroklima und damit auf das Wohlbefinden der Menschen sowie auch die biologische Vielfalt hat und Gärten das lokale Klima über ihre Grenzen hinaus positiv beeinflussen. Entsprechende Modellrechnungen bieten damit eine Grundlage für die Modifikation der Bestandsstruktur, um die mikro- bzw. lokalklimatischen Wirkungen der Gärten zu optimieren.

Hieraus lassen sich für den Schutz der naturräumlichen Funktionen historischer Gärten vor den Folgen des Klimawandels die folgenden Empfehlungen ableiten:

- Zu den Substrateigenschaften - insbesondere auch in tieferen Bodenschichten sowie zur Heterogenität der Substrate existieren für historische Gärten in der Regel keine flächendeckenden Erhebungen. Daher wird für alle historischen Gärten eine systematische Bodenkartierung empfohlen, um die Standortpotentiale differenziert 
bewerten und die daraus resultierenden Erhaltungsmaßnahmen standortspezifisch durchführen zu können. Darüber hinaus sollte ein Stoffstrom-Management implementiert werden, um Nährstoffentzüge und die Humusentwicklung räumlich differenziert einschätzen und bedarfsgerechte Ausgleichsmaßnahmen treffen zu können. Dies kann über ein betriebsinternes Recycling, aber auch durch Düngungsmaßnahmen realisiert werden. Mit Blick auf die Steigerung des Humusgehaltes sollten Mulch-Konzepte konsequent umgesetzt und Bodensubstrate bei Baumpflanzungen mit stabiler organischer Substanz angereichert werden, um die Wasser- und Nährstoffhaltekapazität im Wurzelraum zu steigern. Maßnahmen der Bodenmelioration sollten hierbei durch gezielte periodische ernährungskundliche Analysen ergänzt werden.

- Der Erhalt der genetischen Vielfalt bildet die Grundlage für die Anpassung der Vegetation an den Klimawandel. Neuere genetische Analysemethoden gewähren einen Einblick in die ursprüngliche Herkunftsregion und liefern damit einen Hinweis für die potenzielle Resilienz des Pflanzenmaterials. Die Anwendung entsprechender Analysen kann daher zukünftige Entscheidungen zur Auswahl von Saat- und Pflanzgut unterstützen. Dies gilt in ganz besonderer Weise für die Charakterisierung von Saatund Pflanzgut aus eigener Anzucht. Daher wird empfohlen, in parkeigenen Wirtschaftsbetrieben und Baumschulen entsprechende genetische Analysen zu nutzen, um die klimatische Anpassungsfähigkeit des Pflanzmaterials einschätzen zu können.

- Analog zur pflanzlichen Vielfalt kann die Ausbreitung von Tierarten für die Erhaltung der Gärten ein zunehmendes Problem darstellen. Die Einwanderung neuer Tierarten (Neozoen) ist ganz wesentlich durch den Klimawandel verursacht, aber auch die Internationalisierung des Handels und die Zunahme des Tourismus spielen hierbei eine Rolle. Mit Hilfe von Modellen lässt sich die generelle Verbreitungsdynamik sehr gut abschätzen. Ein Monitoring zur regionalspezifischen oder lokalen Ausprägung dieses Phänomens könnte daher die Lösung solcher Probleme in einem wichtigen Bereich unterstützen.

- Der Schutz historischer Gärten vor den Folgen des Klimawandels ist ohne ein vorausschauendes Erhaltungsmanagement (Vorsorgeprinzip) nicht möglich. Neuartige Sensorik und die damit verbundenen Technologien der Digitalisierung bieten hier vielfältige Optionen, relevante Zustandsdaten zeitnah und georeferenziert aufzunehmen und so denkmalpflegerische Entscheidungen zeitlich und räumlich differenziert treffen zu können. Gerade im Hinblick auf Extremereignisse kann der Einsatz innovativer Technologien einen großen Beitrag für die Vermeidung oder Begrenzung von Schadeffekten leisten.

- Eine zentrale Empfehlung der IAG lautet daher, die Digitalisierung und moderne Sensorik (z.B. Bodenwassergehalt, Kohlenstoffgehalt, Nährstoffgehalte) und Visualisierungsinstrumente zu nutzen, um Bewirtschaftungsmaßnahmen zeit- und bedarfsgerecht durchführen und gleichzeitig dokumentieren zu können. Wünschenswert 
wäre, ein solches System mit historischen Daten zu ergänzen und im Sinne der Gartendenkmalpflege und Kunstgeschichte zu einem Gartenarchiv weiterzuentwickeln. Langfristig ließen sich hieraus Ansätze für künstliche Intelligenz entwickeln, die auf Grundlage sensorischer Netzwerke die Entwicklung selbstlernender Systeme für die Prävention und den Schutz vor Risiken ermöglichen.

\section{Historische Gärten als Gegenstand der Sozialwissenschaften}

Historische Gärten, Parks und Kulturlandschaften sind als Kunstwerke und besondere Naturräume letztlich immer in einen gesellschaftlichen Zusammenhang eingebettet, denn bis heute werden sie von Menschen genutzt, geschätzt und verwaltet. Daher hat ihre gesellschaftliche Einbettung mit unterschiedlichsten Nutzungsansprüchen auch einen Einfluss auf die Resilienz historischer Gärten gegenüber dem Klimawandel. Es empfiehlt sich folglich für die Gartenverantwortlichen, die Wünsche und Bedürfnisse der verschiedenen Nutzergruppen (u.a. Touristinnen und Touristen, Anwohnerinnen und Anwohner) wahrzunehmen und proaktiv in Entscheidungen über Maßnahmen einzubinden.

Die für historische Gärten Verantwortlichen stehen insgesamt stets vor der Aufgabe, Strategien zur Mobilisierung ökonomischer Ressourcen, gesellschaftlicher Legitimation, politischer Durchsetzungskraft oder zum Umgang mit lokalen Freundes- und Unterstützerkreisen zu entwickeln. Doch ihre Perspektiven auf den Klimawandel sind, so ein Ergebnis der IAG, durchaus nicht eindeutig, da sie die Folgen desselben unterschiedlich bewerten und teilweise sogar für ein zu vernachlässigendes Thema halten. Dies deutet nicht nur Möglichkeiten, sondern auch Grenzen im Umgang mit gesellschaftlichen Rahmenbedingungen in historischen Gärten an. Soziologische Untersuchungen zum organisationalen Umfeld sollten daher jeweils gezielt auf den einzelnen Garten abgestimmt sein.

Künftige Forschungen zu den historischen Gärten sollten die sozialwissenschaftliche Expertise als dritte Säule neben den Kultur- und Naturwissenschaften sichtbarer einbeziehen, um ein ganzheitliches Bild zu gewinnen. Denn erst eine auf die jeweiligen Adressatenkreise gut abgestimmte Vermittlungsstrategie, die auf einer umfassend angelegten Zusammenschau des Wissens basiert, ermöglicht es Politik und Gesellschaft, künftig deutlicher Verantwortung zu übernehmen und Rahmenbedingungen zu schaffen, unter denen historische Gärten weiterhin identitätsstiftend, gesundheitsfördernd und vielseitig bildend wirken können.

Angesichts des Klimawandels ist aus sozialwissenschaftlicher Perspektive zur Bewahrung historischer Gärten Folgendes erforderlich:

- Es sollte Wert darauf gelegt werden, die bestehenden Governance-Strukturen weiter zu verbessern bzw. auf ihre Eignung hin zu überprüfen, um insbesondere auch den vielfältigen Einbettungen von Gärten in ihr gesellschaftliches und politisches Umfeld gerecht zu werden. Ohne passende Strukturen - als Beispiel sei das Fehlen eines Gar- 
tendirektorats genannt - kann weder Gartendenkmalpflege betrieben werden noch ist an eine Beeinflussung des Nutzungsverhaltens von Besucherinnen und Besuchern zu denken.

- Die Potentiale einer soziologischen Rahmung und ökonomischen Aufwertung historischer Gärten ließen sich künftig besser nutzen, indem Erfahrungen mit dem Konzept der Ökosystemleistungen aus dem Naturschutz reflektiert werden.

- Konkrete Fallstudien der IAG geben erste Hinweise, wie geändertes gesellschaftliches Anspruchsverhalten und der aus Klimaszenarien resultierende Schutzanspruch mit denkmalpflegerischer Bewahrung in Einklang zu bringen sein könnten: Eine Bedeutungsverschiebung von der Wahrnehmung eines historischen Gartens als Ort für den Freizeitaufenthalt hin zur Betonung seines Denkmalstatus' kann dazu führen, neues Potential für verstärkte (ideelle wie auch finanzielle) Unterstützung und für eine neue Wertschätzung durch die Bevölkerung zu gewinnen.

- Zur Vermeidung von Nutzungskonflikten, die vielerorts zwischen Freizeitansprüchen und Vorgaben aus dem Status des Gartens als denkmalgeschütztes Kulturgut bestehen, ist eine Steuerung des Besucherverhaltens, unter anderem durch eindeutig abgefasste und verständliche Parkordnungen sowie darüber hinausgehende verhaltenslenkende Maßnahmen, unabdingbar.

- Historische Gärten sind bestens geeignete Orte, um Narrative, die den Klimawandel als Aspekt der Geschichte und Erfahrung der Gegenwart zugleich aufgreifen, zu entwickeln. Entsprechende Vermittlungsangebote können zu einem Wandel des Naturverständnisses und zu einem besseren Wissen über den Wert und die Bedeutung historischer Gärten beitragen. Über solche Angebote kann auch vermittelt werden, mit welchem Aufwand der Erhalt solcher Gärten verbunden ist.

- Eine weitere Empfehlung lautet daher, die Vielfältigkeit der Nutzwirkungen, die von diesen Gärten ausgehen, stärker in die öffentliche Wahrnehmung zu rücken. Gleichzeitig sollten in den Gärten freie Räume für Rekreation geschaffen und die Nutzungen entsprechend gelenkt werden, um sensible Bereiche zu entlasten.

- Letztlich bieten die Gärten als Archive ein erhebliches Wissenspotenzial. Gerade der kontinuierliche Kontakt zu jungen Menschen erscheint hier geeignet, eine nachhaltig wirksame Grundlage für die Wertschätzung historischer Gärten aufzubauen. Dazu ließen sich Erfahrungen aus dem Theaterwesen mit geeigneten Formaten künftig integrieren. Eine höhere Präsenz des Fachpersonals dürfte auch die Hemmschwelle gegen Fehlnutzung erhöhen. Vor allem aber kann dieses Personal eine wichtige Funktion für die Wissensvermittlung erfüllen und damit einen entscheidenden Beitrag für die Wertschätzung der Gärten leisten. Die Gärten sollten daher einen viel umfassenderen Bildungsauftrag erhalten und damit ihrer Funktion als gesellschaftshistorische Identifikationspunkte noch stärker gerecht werden. 
- Eine Umsetzung der vorgenannten Empfehlungen ist in aller Regel nur über zusätzliche Investitionen realisierbar. Die Bereitschaft, entsprechende finanzielle Mittel einzusetzen, wird nur dann entstehen können, wenn für historische Gärten in Gesellschaft und Politik eine angemessene Wertschätzung besteht.

\section{Schlussbemerkungen und Ausblick}

Abschließend ist festzuhalten, dass es stets erforderlich ist, für jeden historischen Garten individuell die komplexen inter- und transdisziplinär verwobenen Prozesse zu erfassen, die seit seiner Entstehung auf ihn gewirkt haben bzw. wirksam sind. Nur auf der Grundlage dieses breit angelegten Wissens kann ein Erhalt oder eine Anpassung angesichts der klimatischen Veränderungen gelingen.

Mit Blick auf die etwa 35.000 Gartendenkmale in Deutschland bedarf es neben kunsthistorischen Grundlagen (Parkpflegewerke) als zweite und dritte Säule des Wissens umfassender natur- und sozialwissenschaftlicher Expertisen in Forschung und Lehre. Die vielfältigen, teilweise neuen Bedürfnisse und Anforderungen, die auf historische Gärten angesichts des Klimawandels zukommen, ließen sich durch diese gebündelten Expertisen erfüllen.

Angesichts des Klimawandels ist es dringend erforderlich, historische Gärten als Kulturdenkmale noch umfassender als Forschungsgegenstand zu begreifen. Ohne wissenschaftliche Begleitung und stetige Forschung zu den oben aufgelisteten Themenfeldern können keine nachhaltigen Erhaltungs-, Anpassungs- oder Restaurierungsmaßnahmen seitens der Gartendenkmalpflege entworfen werden. Eine in diesem umfassenden Sinne wissenschaftsbasierte Gartendenkmalpflege kann beispielgebend für andere Bereiche des Kulturerbes sein. Eine publizistische Aufarbeitung und Dokumentation des Kulturdenkmalbestandes ist gut beraten, hier anzusetzen und Grundlagenwissen zu vermitteln und darüber hinaus eine öffentlich wirksame Darstellung der vielfältigen Bedeutungen und Funktionen der Gartenkunstwerke, einschließlich ihrer Sinngebungs- und Identifikationsangebote für die heutige Gesellschaft, zu leisten. 